Topological Methods in Nonlinear Analysis

Volume 47 , No. 1, 2016, 55-72

DOI: $10.12775 /$ TMNA.2015.087

(C) 2016 Juliusz Schauder Centre for Nonlinear Studies

Nicolaus Copernicus University

\title{
PERIODIC SOLUTIONS OF SINGULAR EQUATIONS
}

\author{
Antonio J. UReÑA
}

\begin{abstract}
We study second-order ordinary differential equations of Newtonian type. The forcing terms under consideration are the product of a nonlinearity which is singular at the origin with an indefinite weight. Under some additional assumptions we show the existence of periodic solutions.
\end{abstract}

\section{Introduction and main result}

The purpose of this note is to study the solvability of the $T$-periodic problem associated to the equation

$$
\ddot{x}=h(t) g(x), \quad x>0,
$$

under the following assumptions: $T>0$ is a fixed period and

$\left(\mathrm{h}_{0}\right)$ the weight function $h: \mathbb{R} \rightarrow \mathbb{R}$ is $T$-periodic and locally integrable,

(g) the nonlinearity $g:] 0,+\infty[\rightarrow] 0,+\infty[$ is a decreasing homeomorphism.

In particular, at $x=0$ there is a singularity, and we are interested in $T$-periodic solutions $x=x(t)$ which avoid this singularity in the sense that $x(t)>0$ for all $t \in[0, T]$.

2010 Mathematics Subject Classification. 34B15, 34B16.

Key words and phrases. Periodic solutions; singular equations; indefinite weight.

Supported by project MTM2011-23652, Ministerio de Educación y Ciencia, Spain, and FQM2216, Junta de Andalucía. 
As a model, we have in mind the so-called generalized Emden-Fowler equation with negative exponent $-p$,

$$
\ddot{x}=\frac{h(t)}{x^{p}},
$$

corresponding to the choice $g(x)=1 / x^{p}$ (here $p$ is a positive constant). Periodic solutions of equations of this type appear in some problems of engineering, including the stabilization of matter-wave breathers in Bose-Einstein condensates, the propagation of guided waves in optical fibres, or the electromagnetic trapping of a neutral atom near a charged wire [4]. In the case of $p=2$, this equation also is used to model the one-dimensional oscillations of a free $\alpha$-particle subject to the influence of the electric field created by a charge of a time-depending magnitude fixed at the origin (magnetic interaction between the charges is excluded).

The Dirichlet problem associated to (1.2) has been treated by many authors. To the best of our knowledge, the history starts with Nachman and Callegari [14], who considered the particular case $p=1$ and $h(t)=-t$. A mayor breakthrough came with the work of Taliaferro [16], who gave necessary and sufficient conditions on the (negative but otherwise arbitrary) weight function $h$ for the solvability of this problem. This paper received a lot of interest and was subsequently generalized in different directions by Luning and Perry [13], Bobisud, O'Regan and Royalty [2], Gatica, Oliker and Waltman [7], Guo [10], Janus and Myjak [12], Habets and Zanolin [11], and Gaudenzi, Habets and Zanolin [8], among others. See, e.g. [1] for a classical review on the subject.

Contrastingly, the corresponding periodic problem seems to have received little attention in the literature, except for the pioneer work by Bravo and Torres [4]. When $p=3$ and the weight function $h$ is piecewise-constant with only two values, they used a careful phase-plane analysis to prove that (1.2) has a periodic solution if and only if the two values of $h$ have different sign and the integral of $h$ over a period is negative. The question arises: how do these results extend to equation (1.1) under more general assumptions on $h$ and $g$ ?

It suffices to integrate both sides of (1.1) to obtain the first necessary condition for the existence of a $T$-periodic solution: the weight function $h$ (assumed nontrivial) should change sign (remember that the nonlinearity $g$ is positive). It means that we are dealing with indefinite problems. In particular,

$\left(\mathrm{h}_{1}\right) \operatorname{meas}\{t \in[0, T]: h(t)>0\}>0$.

On the other hand, after dividing both sides of the equation by $g$ and integrating by parts the left side, one obtains that, at least when $g:] 0,+\infty[\rightarrow] 0,+\infty[$ is a $C^{1}$-diffeomorphism, the second necessary condition for a $T$-periodic solution to exist is that

$$
\left(\mathrm{h}_{2}\right) \frac{1}{T} \int_{0}^{T} h(t) d t<0
$$


Our previous question can now be made more precise: what additional conditions on the nonlinearity $g$ and the weight function $h$ can be added to $(\mathrm{g})$ and $\left(\mathrm{h}_{0}\right)-\left(\mathrm{h}_{2}\right)$ in order to ensure the existence of a T-periodic solution of (1.1)?

We shall focus our attention on two families of weight functions: the class of piecewise-constant functions and the class of functions having only nondegenerate zeroes. The (discontinuous) T-periodic function $h: \mathbb{R} \rightarrow \mathbb{R}$ is said to be piecewiseconstant if there is a partition $0=t_{0}<t_{1}<\ldots<t_{p}=T$ of $[0, T]$ such that $\left.h\right|_{] t_{i-1}, t_{i}[} \equiv h_{i}$ is constant for each $i=1, \ldots, p$. On the other hand, the continuous and $T$-periodic function $h$ is said to have only nondegenerate zeroes provided that it is continuously differentiable, with nonvanishing derivative, in a neighbourhood of each of its zeroes.

Our assumptions on the nonlinearity $g$ will consist of making more precise the strength of the singularity at $x=0$. With this purpose we shall say that this singularity is strong (resp. very strong) provided that

$$
\int_{0}^{1} g(x) d x=+\infty \quad\left(\operatorname{resp} . \quad \int_{0}^{1} x g(x) d x=+\infty\right) .
$$

Combining the assumptions on $h$ with those on $g$ we shall distinguish two possible situations:

(A) The weight function $h$ is piecewise constant and the singularity of $g$ at $x=0$ is strong.

(B) The weight function $h$ has only nondegenerate zeroes and the singularity of $g$ at $x=0$ is very strong.

The main result of this paper is the following:

Theorem 1.1. Assume $(\mathrm{g})$ and $\left(\mathrm{h}_{0}\right)-\left(\mathrm{h}_{2}\right)$. Assume further that we are either in situation (A) or in situation (B). Then (1.1) has a T-periodic solution.

Some comments on the definition of strong singularity considered above: In [9], Gordon introduced a notion of 'strong force' which has become very popular. Adapting his definition to our situation, the nonlinearity $g$ is a strong force provided that there exist some $\varepsilon>0$ and a $C^{2}$ function $\left.\varphi:\right] 0, \varepsilon[\rightarrow \mathbb{R}$ with $\varphi(x) \rightarrow+\infty$ as $x \rightarrow 0$ such that the 'potential energy' function $\mathscr{G}(x):=$ $-\int_{1}^{x} g(y) d y$ satisfies $\mathscr{G}(x) \geq \varphi^{\prime}(x)^{2}$ for every $x>0$. It is easy to check that this implies that $\int_{0}^{1} \mathscr{G}(x) d x=+\infty$, and, integrating by parts, $\int_{0}^{1} x g(x) d x=+\infty$. Thus, Gordon's notion of strong force actually implies that the singularity of $g$ at $x=0$ is very strong in the sense considered above. The converse does not hold; it suffices to check that the model nonlinearity $g(x)=1 / x^{p}$ has a strong singularity at $x=0$ provided that $p \geq 1$, and a very strong singularity at $x=0$ provided that $p \geq 2$, while it is a strong force in the sense of Gordon when $p \geq 3$.

The related Neumann problem has been recently studied by Boscaggin and Zanolin [3]. They introduce a suitable change of variables and subsequently look 
for the intersection points between the curves of solutions departing or arriving with zero velocity. In this way, they show the existence of Neumann solutions in many cases where the weight function changes sign once on the given time interval. While their results apply to a much wider range of nonlinearities $g$ than those considered in the present paper, it looks likely that the arguments which follow can be adapted to prove the existence of Neumann solutions in some situations when the weight function changes sign an arbitrary number of times.

Theorem 1.1 motivates the question of the necessity of its various assumptions. For instance, it seems natural to ask whether the result still holds if the only-nondegenerate-zeroes assumption is removed from situation (B), or when the condition on the strength of the singularity is removed from situations (A) and (B). In a forthcoming paper [15] it will be shown that the answer to both questions is negative.

This paper is structured in the following way. In Section 2 we consider the semiperiodic problem, i.e. we look for solutions $x:[0, T] \rightarrow \mathbb{R}$ of our equation satisfying $x(0)=x(T)$, but with no condition on the slopes $\dot{x}(0), \dot{x}(T)$. This less restrictive problem is underdetermined, and continuation methods from degree theory imply the existence of a continuum $\Sigma$ of solutions containing curves with any positive minimum value. Next, we observe that some elements $x=x(t)$ of $\Sigma$ with large minimum value satisfy $\dot{x}(T) \leq \dot{x}(0)$. In Section 3 we see that the solutions of the semiperiodic problem satisfying this property have a bounded oscillation on the time interval $[0, T]$. A partial converse of this result is established in Section 4, where we observe that the solutions whose minimum value is small have a large oscillation. It means that $\Sigma$ must contain also some curves $x=x(t)$ with $\dot{x}(T) \geq x(0)$, and being connected, it must contain a solution of the semiperiodic problem with $\dot{x}(0)=\dot{x}(T)$. This is a $T$-periodic solution of our equation, and Theorem 1.1 follows.

\section{The semiperiodic problem}

Throughout this section we assume $\left(\mathrm{h}_{0}\right)-\left(\mathrm{h}_{2}\right)$ and $(\mathrm{g})$. Let us momentarily weaken the periodic boundary conditions and consider firstly an underdetermined problem, which we shall call (SP) (the initials coming from 'semiperiodic problem'). It consists of equation (1.1) and the boundary condition

$$
x(0)=x(T) .
$$

Solutions of (SP) may not give rise to periodic solutions of (1.1) because the derivatives at times $t=0$ and $t=T$ may be different. Problem (SP), being less restrictive, should be easier to solve than the full periodic problem. Indeed, the main result of this section, which will be obtained from continuation arguments of degree theory, states that (SP) has many solutions. 
Proposition 2.1. There exists a connected set $\Sigma \subset C^{1}[0, T]$ of solutions of (SP) satisfying

$$
\left.\left\{\min _{[0, T]} x: x \in \Sigma\right\}=\right] 0,+\infty[.
$$

We shall prepare the proof of this result by means of some lemmas. The first one exploits the fact that the right hand side of our equation becomes weaker and weaker as we approach the infinity to give a priori bounds for the oscillation of solutions of (1.1) (not necessarily satisfying any boundary condition), provided that the derivative vanishes at some time when the solution is far away from the origin.

Lemma 2.2. There exists some $M>1$ with the following property: any solution $x=x(t)$ of (1.1) having a critical value $x_{c} \geq M$ is defined on the whole time interval $[0, T]$ and satisfies $\left|x(t)-x_{c}\right|<1$ for all $t \in[0, T]$.

Proof. We let $M:=g^{-1}\left(1 /\left(T\|h\|_{L^{1}(0, T)}\right)\right)+1$ and choose a solution $x=x(t)$ of our equation with $x\left(t_{c}\right)=x_{c} \geq M$ and $\dot{x}\left(t_{c}\right)=0$ for some $t_{c} \in[0, T]$. The reasoning for $t \in\left[0, t_{c}\right]$ being analogous, we can limit ourselves to establish the result for $t \in\left[t_{c}, T\right]$. With this purpose we use a contradiction argument and assume that there is some $\left.\left.t_{1} \in\right] t_{c}, T\right]$ such that $\left|x(t)-x\left(t_{c}\right)\right|<1$ for every $t \in\left[t_{c}, t_{1}\left[\right.\right.$ and $\left|x\left(t_{1}\right)-x\left(t_{c}\right)\right|=1$. Now, $x(s)>g^{-1}\left(1 /\left(T\|h\|_{L^{1}(0, T)}\right)\right)$, or, what is the same, $0<g(x(s))<1 /\left(T\|h\|_{L^{1}(0, T)}\right)$ for any $s \in\left[t_{c}, t_{1}[\right.$. It follows that

$$
|\dot{x}(t)|=\left|\dot{x}(t)-\dot{x}\left(t_{c}\right)\right|=\left|\int_{t_{c}}^{t} h(s) g(x(s)) d s\right|<\frac{1}{T} \quad \text { for any } t \in\left[t_{c}, t_{1}\right],
$$

and integrating again we deduce that $\left|x\left(t_{1}\right)-x\left(t_{c}\right)\right| \leq \int_{t_{c}}^{t_{1}}|\dot{x}(t)| d t<1$. This assertion contradicts the choice of $t_{1}$ and concludes the proof.

From now on we fix $M>1$ as given by Lemma 2.2 and define $\alpha_{0}:=M+1$. Choose some number $\beta_{0} \geq M+3$, and pick solutions $\alpha=\alpha(t), \beta=\beta(t)$ of (1.1) satisfying the initial condition

$$
x(0)=\gamma, \quad \dot{x}(0)=0,
$$

with $\gamma=\alpha_{0}$ and $\gamma=\beta_{0}$, respectively. Lemma 2.2 gives

$$
\alpha(t)<M+2 \leq \beta_{0}-1<\beta(t) \text { for any } t \in[0, T],
$$

so that, for any $x_{0} \in\left[M+2, \beta_{0}-1\right]$, the functions $\alpha, \beta$ make up an ordered pair of lower and upper solutions for the Dirichlet problem (SP) $x_{x_{0}}$, consisting in combining equation (1.1) with the initial condition

$$
x(0)=x(T)=x_{0} .
$$

Consequently, the usual upper and lower solutions method for the Dirichlet problem (see, e.g. [6]) implies that (SP) $)_{x_{0}}$ is solvable. The arbitrariness of $\beta_{0} \geq M+3$ 
means that this is true for every $x_{0} \geq M+2$, and we deduce the existence of infinitely many solutions of (SP).

With the purpose of proving Proposition 2.1 we need to refine this argument and construct large connected sets of solutions of (SP). We start by observing that, again by Lemma 2.2 , if $\beta_{0} \geq M+5$, then for every $x_{0} \in\left[M+3, \beta_{0}-2\right]$ and any solution $x=x(t)$ of $(\mathrm{SP})_{x_{0}}$ with $\alpha(t) \leq x(t) \leq \beta(t)$ on $[0, T]$ one has

$$
\alpha(t)<M+2 \leq x_{0}-1<x(t)<x_{0}+1 \leq \beta_{0}-1<\beta(t)
$$

for every $t \in[0, T]$, the fact which will be used later.

We now rewrite our problem as a fixed-point equation depending on a parameter. This is a standard procedure, so that we describe it briefly. We shall work on the Banach space $X:=C(\mathbb{R} / T \mathbb{Z})$ of continuous and $T$-periodic functions on the real line. This space splits as the direct sum of the 1-dimensional subspace of constant functions, canonically identified to $\mathbb{R}$, and the hyperplane $\widetilde{X}:=\{x \in X: x(0)=0\}$. Correspondingly, we decompose functions $x \in X$ in the form $x=x_{0}+\widetilde{x}$, where $x_{0}=x(0)$ and $\widetilde{x} \in \widetilde{X}$. Problem (SP) $x_{x_{0}}$ now becomes a fixed point equation in $\widetilde{X}$, depending on the parameter $x_{0}$ :

$$
\widetilde{x}=F\left[x_{0}, \widetilde{x}\right]:=K N\left[x_{0}, \widetilde{x}\right] .
$$

Here, $N\left[x_{0}, \widetilde{x}\right](t)=h(t) g\left(x_{0}+\widetilde{x}(t)\right)$ and $(K x)(t):=\int_{0}^{T} G(t, s) x(s) d s$ are, respectively, the Nemytskil operator associated to the right hand side of our equation, and the integral operator whose kernel $G=G(t, s)$ is the Green function for the 1-dimensional Poisson equation $\ddot{u}=\varphi(t)$ under homogeneous Dirichlet boundary conditions. Equation (2.3) makes sense on the set

$$
\Omega:=\left\{\left(x_{0}, \widetilde{x}\right) \in\right] 0,+\infty\left[\times \widetilde{X}: x_{0}+\widetilde{x}(t)>0 \text { for any } t \subset \mathbb{R}\right\},
$$

which is open in $\mathbb{R} \times \widetilde{X}$. Observe that $N: \Omega \rightarrow L^{1}(\mathbb{R} / T \mathbb{Z})$ is continuous, while $K: L^{1}(\mathbb{R} / T \mathbb{Z}) \rightarrow \widetilde{X}$ is linear and compact. Moreover, $N$ takes closed subsets of $\mathbb{R} \times \widetilde{X}$ which are contained in $\Omega$ into bounded subsets of $L^{1}(\mathbb{R} / T \mathbb{Z})$. In this way, $F:=K \circ N: \Omega \rightarrow \widetilde{X}$ is completely continuous, and the Leray-Schauder degree theory applies.

We let, as before, $\alpha_{0}:=M+1$, and fix some number $\beta_{0} \geq \alpha_{0}+5$, together with corresponding solutions $\alpha=\alpha(t), \beta=\beta(t)$ of $(I)_{\alpha_{0}}$ and $(I)_{\beta_{0}}$, respectively. Consider the set

$$
\begin{aligned}
\Omega_{*}:=\left\{\left(x_{0}, \tilde{x}\right) \in \mathbb{R} \times \widetilde{X}: M+\right. & \leq x_{0} \leq \beta_{0}-2 \\
& \text { and } \left.\alpha(t)<x_{0}+\widetilde{x}(t)<\beta(t) \text { for all } t \in[0, T]\right\},
\end{aligned}
$$

and observe that $\Omega_{*}$ is bounded and open relative to $\left[\alpha_{0}+2, \beta_{0}-2\right] \times \widetilde{X}$, its closure $\overline{\Omega_{*}}$ being contained in $\Omega$. Moreover, in view of (2.2), no solution $\left(x_{0}, \tilde{x}\right)$ of (2.3) belongs to the boundary $\overline{\Omega_{*}} \backslash \Omega_{*}$. And well-known arguments (see, e.g. [6, Chapter III]) show that, for each $x_{0} \in\left[M+3, \beta_{0}-2\right]$, the Leray-Schauder 
fixed-point degree of $F\left[x_{0}, \cdot\right]$ on the section $\Omega_{*} \cap\left(\left\{x_{0}\right\} \times \tilde{X}\right)$ is one. Combining the excision property of the degree with Lemma 2.2 we see that, indeed,

$$
\operatorname{deg}_{L S}\left(\operatorname{Id}_{\widetilde{X}}-F\left(x_{0}, \cdot\right), \widetilde{\mathbb{B}}, 0\right)=1
$$

(we denote by $\widetilde{\mathbb{B}}$ to the open unit ball on $\widetilde{X}$ ). In principle, this is true for every $x_{0} \in\left[M+3, \beta_{0}-2\right]$, but the arbitrariness of $\beta_{0} \geq M+5$ means that the equality above actually holds for every $x_{0} \geq M+3$.

Proof of Proposition 2.1. In view of the arguments above, it suffices to find a connected set $\Sigma \subset \mathbb{R} \times \widetilde{X}$ of solutions of (2.3) with $\left\{\min _{[0, T]}\left(x_{0}+\widetilde{x}\right)\right.$ : $\left.\left.\left(x_{0}, \widetilde{x}\right) \in \Sigma\right\}=\right] 0,+\infty[$.

Our departing point will be the computation of the degree done in (2.4); reasoning as in [5, pp. 168-169] we find a connected component $\Sigma$ of the set of solutions $\left(x_{0}, \widetilde{x}\right) \in \Omega$ of $(2.3)$ such that $\Sigma \cap\left(\left\{x_{0}\right\} \times \widetilde{\mathbb{B}}\right) \neq \emptyset$ for every $x_{0} \geq M+3$. It follows that the interval $\left\{\min _{t \in[0, T]}\left(x_{0}+\tilde{x}\right):\left(x_{0}, \tilde{x}\right) \in \Sigma\right\}$ contains $[M+3,+\infty[$, so that it only remains to check that

$$
\inf _{\left(x_{0}, \tilde{x}\right) \in \Sigma}\left[\min _{[0, T]}\left(x_{0}+\widetilde{x}\right)\right]=0 .
$$

Standard continuation theorems state that the connected set $\Sigma$, which comes from $[M+3,+\infty[\times \widetilde{\mathbb{B}}$, cannot be entirely contained there, and must instead satisfy one of the following three possibilities:

(i) The projection of $\Sigma$ on $\tilde{X}$ is unbounded in $\tilde{X}$.

(ii) The projection of $\Sigma$ on $\widetilde{X}$ is bounded in $\widetilde{X}$, and $\bar{\Sigma} \cap \partial \Omega \neq \emptyset$. Observe that it includes in particular the possibility that the projection of $\Sigma$ on $\mathbb{R}$ is the whole interval $] 0,+\infty[$.

(iii) The projection of $\Sigma$ on $\widetilde{X}$ is bounded in $\widetilde{X}$, and $\Sigma \cap\left(\left\{x_{0}\right\} \times \widetilde{X}\right) \not \subset\left\{x_{0}\right\} \times \widetilde{\mathbb{B}}$ for every $x_{0} \geq M+3$.

(See Figure 1.) Let us start by showing that case (iii) cannot happen in our problem. Indeed, if one assumes the projection of $\Sigma$ on $\widetilde{X}$ to be bounded, there must exist some $M_{1}>0$ such that $\min _{[0, T]}\left(x_{0}+\widetilde{x}\right)>M$ for every $\left(x_{0}, \widetilde{x}\right) \in \Sigma$ with $x_{0}>M_{1}$. Lemma 2.2 then implies that $\Sigma \cap\left(\left\{x_{0}\right\} \times \widetilde{X}\right) \subset\left\{x_{0}\right\} \times \widetilde{\mathbb{B}}$ if $x_{0}>M_{1}$, contradicting (iii).

Finally, we observe that cases (i) and (ii) imply (2.5). This is obviously true for case (ii), as $\partial \Omega=\left\{\left(x_{0}, \tilde{x}\right) \in\left[0,+\infty\left[\times \widetilde{X}: \min _{[0, T]}\left(x_{0}+\widetilde{x}\right)=0\right\}\right.\right.$. Concerning case (i), we use a contradiction argument and assume instead the existence of some $\rho>0$ such that $x_{0}+\tilde{x} \geq \rho$ for any $t \in[0, T]$. Our Nemytskii operator $N: \Omega \rightarrow L^{1}(\mathbb{R} / T \mathbb{Z})$ is bounded on the set $\Omega_{\rho}:=\left\{\left(x_{0}, \widetilde{x}\right) \in \mathbb{R} \times \widetilde{X}: x_{0}+\widetilde{x}(t) \geq\right.$ $\rho$ for all $t \in[0, T]\}$, and (2.3) implies that the projection of $\Sigma$ on $\tilde{X}$ is bounded. This is a contradiction and concludes the proof. 


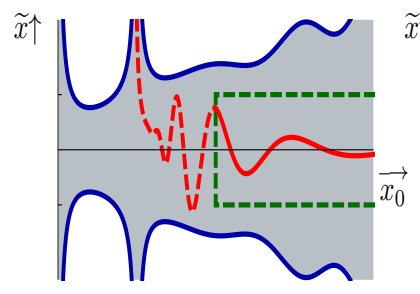

Case (i)

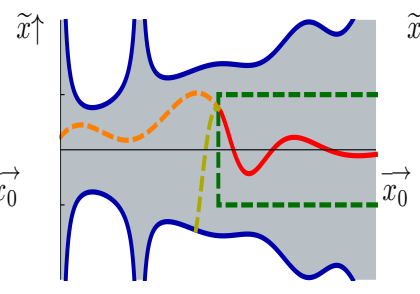

Case (ii)

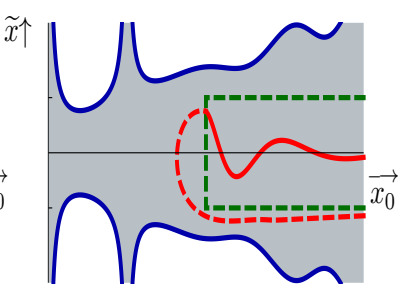

Case (iii)

Figure 1. The possibilities of the set $\Sigma$.

To conclude this section we study the difference of slope of the elements in our connected set $\Sigma$ at the endpoints of the time interval $[0, T]$. We show that the final slope must be smaller than the initial slope, at least for some functions $x \in \Sigma$.

Lemma 2.3. Let the connected set $\Sigma$ be as given by Proposition 2.1. Then it contains some curves $x=x(t)$ with $\dot{x}(T) \leq \dot{x}(0)$.

Proof. It is divided into three steps.

Step 1. There exists a constant $C>0$ (depending only on $h$ and $T$ ), such that every solution $x=x(t)$ of (SP) satisfies

$$
\max _{[0, T]} x-\min _{[0, T]} x \leq C g(\min x) .
$$

Indeed, since $g:] 0,+\infty[\rightarrow] 0,+\infty[$ is decreasing, the Nemytskiu operator $N$ has the following property:

$$
\left\|N\left[x_{0}, \widetilde{x}\right]\right\|_{L^{1}} \leq\|h\|_{L^{1}} g\left(\min _{[0, T]} x\right) .
$$

On the other hand, the operator $K: L^{1}(\mathbb{R} / T \mathbb{Z}) \rightarrow \widetilde{X}$ being continuous, we see that every solution $\left(x_{0}, \widetilde{x}\right)$ of $(2.3)$ satisfies

$$
\|\widetilde{x}\|_{L^{\infty}} \leq\|K\|\|h\|_{L^{1}} g\left(\min _{[0, T]}\left(x_{0}+\widetilde{x}\right)\right) .
$$

Thus, the result follows after taking $C:=2\|K\|\|h\|_{L^{1}}$.

Step 2. There is a constant $0<\varepsilon<1$ with the following property: if $x=x(t)$ is a solution of $(\mathrm{SP})$ with $\dot{x}(0)<\dot{x}(T)$, then $g\left(\max _{[0, T]} x\right)<\varepsilon g\left(\min _{[0, T]} x\right)$.

To check this we integrate both sides of the differential equation, to find

$$
\begin{array}{rl}
0<\dot{x}(T)-\dot{x}(0)=\int_{0}^{T} & h(t) g(x(t)) d t \\
& \quad \leq g\left(\min _{[0, T]} x\right) \int_{0}^{T} h_{+}(t) d t-g\left(\max _{[0, T]} x\right) \int_{0}^{T} h_{-}(t) d t,
\end{array}
$$


where $h=h_{+}-h_{-}, h_{+}:=\max (h, 0)$ and $h_{-}:=\min (-h, 0)$. Consequently, we see that

$$
g\left(\max _{[0, T]} x\right)<\varepsilon g\left(\min _{[0, T]} x\right),
$$

where $\varepsilon:=\int_{0}^{T} h_{+}(t) d t / \int_{0}^{T} h_{-}(t) d t$. Observe that $0<\varepsilon<1$ as claimed (since $\left.\int_{0}^{T} h(t) d t<0\right)$.

Step 3. The end of the proof.

Combining the first and second steps we see that, if $x=x(t)$ is a solution of (SP) with $\dot{x}(0)<\dot{x}(T)$, then $g(\min x+C g(\min x))<\varepsilon g(\min x)$, where $0<\varepsilon<1$ is a constant. We prove the result by a contradiction argument and assume instead that all elements $x \in \Sigma$ satisfy $\dot{x}(0)<\dot{x}(T)$; this yields

$$
g(u+C g(u))<\varepsilon g(u), \quad u>0 .
$$

We consider the increasing sequence $\left\{u_{n}\right\}_{n}$ of positive numbers defined by

$$
u_{0}:=1, \quad u_{n}:=u_{n-1}+C g\left(u_{n-1}\right) \quad \text { if } n \geq 1,
$$

and it follows from (2.6) that $g\left(u_{n}\right)<\varepsilon g\left(u_{n-1}\right)$, and hence,

$$
g\left(u_{n}\right)<\varepsilon^{n} g\left(u_{0}\right) \quad \text { for every } n \geq 1 .
$$

Since $u_{n}=u_{0}+C\left(g\left(u_{0}\right)+g\left(u_{1}\right)+\ldots+g\left(u_{n-1}\right)\right)$ we deduce that $\left\{u_{n}\right\}$ is a convergent sequence, and in view of (2.7), its limit must be a zero of $g$. This is impossible since $g$ was assumed always positive, and the proof is complete.

\section{The oscillation of lower solutions is bounded}

Throughout this section we assume $\left(\mathrm{h}_{0}\right)-\left(\mathrm{h}_{2}\right)$ and $(\mathrm{g})$; furthermore, the singularity of $g$ at $x=0$ will be assumed to be strong. We shall prepare to the proof of Theorem 1.1 with several auxiliary results. To start with, we consider the homeomorphism $\mathcal{G}$ of the interval $[0,+\infty$ [ to itself defined by

$$
\mathcal{G}(x):=\int_{0}^{x} \frac{1}{g(y)} d y, \quad x \geq 0 .
$$

The lemma below gives bounds for the distance between points in $[0,+\infty[$ provided that the distance between their images is controlled.

Lemma 3.1. For any $M>0$ there exists some $N>0$ such that whenever $x, y \geq 0$ satisfy $|\mathcal{G}(x)-\mathcal{G}(y)| \leq M$ then $|x-y| \leq N$.

Proof. Using a contradiction argument, assume the existence of sequences $\left\{x_{n}\right\}_{n},\left\{y_{n}\right\}_{n} \subset[0,+\infty[$ such that

(a) $\left|x_{n}-y_{n}\right| \rightarrow \infty$

(b) $\sup \left|\mathcal{G}\left(x_{n}\right)-\mathcal{G}\left(y_{n}\right)\right|<\infty$. 
The first condition implies that either $\left\{x_{n}\right\}_{n}$ or $\left\{y_{n}\right\}_{n}$ (or both) are unbounded, and after passing to a subsequence we may assume that, for instance, $x_{n} \rightarrow+\infty$. Thus, $\mathcal{G}\left(x_{n}\right) \rightarrow+\infty$, and (b) implies that also $\mathcal{G}\left(y_{n}\right) \rightarrow+\infty$, or, what is the same, $y_{n} \rightarrow+\infty$.

In particular, for $n$ big enough, $x_{n}, y_{n} \geq g^{-1}(1)$. For such values of $n$ one has

$$
\left|\mathcal{G}\left(y_{n}\right)-\mathcal{G}\left(x_{n}\right)\right|=\left|\int_{x_{n}}^{y_{n}} \frac{1}{g(z)} d z\right| \geq\left|y_{n}-x_{n}\right|,
$$

and, remembering (a), we deduce that $\left|\mathcal{G}\left(y_{n}\right)-\mathcal{G}\left(x_{n}\right)\right| \rightarrow+\infty$. This contradicts (b) and concludes the proof of the lemma.

It will be convenient to single out those solutions of (SP) which exit the time interval $[0, T]$ with a smaller slope that they entered it. With this aim we consider problem (LP) consisting of equation (1.1) and the boundary conditions

$$
x(0)=x(T), \quad \dot{x}(0) \geq \dot{x}(T) .
$$

The name of problem (LP) comes from the fact that its solutions are lower solutions for the associated periodic problem. Lemma 2.3 can now be reformulated as follows: the connected set $\Sigma$ given by Proposition 2.1 contains some curves which are solutions of (LP). Our strategy to prove Theorem 1.1 will consist in showing that $\Sigma$ cannot be entirely composed of solutions of (LP) (and hence it contains some periodic solutions), because the oscillation (meaning the difference between the maximum and the minimum values) of the solutions of (LP) is bounded, and it forces the set of such solutions to be uniformly bounded from below by a positive constant. The remaining of the paper is devoted to checking og these facts.

Lemma 3.2. There exists a constant $N>0$ (depending on $h$ and $g$ but not on $x$ ) with the following property: every solution $x=x(t)$ of (LP) satisfies $\max _{[0, T]} x-\min _{[0, T]} x<N$.

ProOF. In view of Lemma 3.1 it suffices to show that every solution $x=x(t)$ of (LP) satisfies

$$
\left|\mathcal{G}\left(x\left(t_{2}\right)\right)-\mathcal{G}\left(x\left(t_{1}\right)\right)\right| \leq 2\|h\|_{L^{1}} \text { for every } t_{1}, t_{2} \in[0, T],
$$

the function $\mathcal{G}$ being defined as in (3.1). This task will be divided into two steps.

Step 1. Assume firstly that $g:] 0,+\infty[\rightarrow] 0,+\infty[$ is a decreasing diffeomorphism of class $C^{1}$. Then, the function $t \mapsto \dot{x}(t) / g(x(t))$ is continuously differentiable on $[0, T]$, and

$$
\frac{d}{d t}\left[\frac{\dot{x}(t)}{g(x(t))}\right]=h(t)-\frac{g^{\prime}(x(t)) \dot{x}(t)^{2}}{g(x(t))^{2}} .
$$


We integrate both sides of the equality, to find that

$-\int_{0}^{T} \frac{g^{\prime}(x(t)) \dot{x}(t)^{2}}{g(x(t))^{2}} d t=\frac{\dot{x}(T)}{g(x(T))}-\frac{\dot{x}(0)}{g(x(0))}-\int_{0}^{T} h(t) d t \leq-\int_{0}^{T} h(t) d t \leq\|h\|_{L^{1}}$, the first inequality follows from the boundary conditions in (LP). Since $g^{\prime}$ is always negative, the expression inside the left integral has constant negative sign, and we deduce

$$
\left\|\frac{g^{\prime}(x) \dot{x}^{2}}{g(x)^{2}}\right\|_{L^{1}} \leq\|h\|_{L^{1}}
$$

and then, going back to (3.3), the triangle inequality gives

$$
\left\|\frac{d}{d t}\left[\frac{\dot{x}}{g(x)}\right]\right\|_{L^{1}} \leq 2\|h\|_{L^{1}}
$$

Moreover, $\dot{x}(t)$ must vanish at some point $t \in[0, T]$ (by Rolle's Theorem), and we conclude

$$
\left\|\frac{\dot{x}}{g(x)}\right\|_{L^{\infty}}=\left\|\frac{d}{d t}[\mathcal{G}(x)]\right\|_{L^{\infty}} \leq 2\|h\|_{L^{1}}
$$

implying (3.2).

Step 2. Let us now go into the general case in which $g$ is a (not necessarily differentiable) decreasing homeomorphism of $] 0,+\infty[$ to itself. Fix a solution $x=x(t)$ of $(\mathrm{LP})$; it is easy to construct, for any $\varepsilon>0$, a $C^{1}$ diffeomorphism $\left.g_{\varepsilon}:\right] 0,+\infty[\rightarrow] 0,+\infty[$ such that

$$
\left(\frac{1}{1+\varepsilon}\right) g(y)<g_{\varepsilon}(y)<(1+\varepsilon) g(y) \quad \text { if } y \in\left[\min _{[0, T]} x, \max _{[0, T]} x\right] .
$$

We are led to consider the modified weight function $h_{\varepsilon} \in L^{1}(0, T)$ defined by $h_{\varepsilon}(t):=g(x(t)) h(t) / g_{\varepsilon}(x(t))$. It satisfies

$$
-(1+\varepsilon)|h(t)|<h_{\varepsilon}(t)<(1+\varepsilon)|h(t)|, \quad t \in[0, T],
$$

and we observe that $x=x(t)$ is a solution of

$$
\ddot{x}(t)=h_{\varepsilon}(t) g_{\varepsilon}(x(t)), \quad t \in[0, T],
$$

for any $\varepsilon>0$. Since $g_{\varepsilon}$ is a $C^{1}$ diffeomorphism of $] 0,+\infty[$ the first step applies, and gives

$$
\left|\mathcal{G}_{\varepsilon}\left(x\left(t_{2}\right)\right)-\mathcal{G}_{\varepsilon}\left(x\left(t_{1}\right)\right)\right| \leq 2\left\|h_{\varepsilon}\right\|_{L^{1}} \leq 2(1+\varepsilon)\|h\|_{L^{1}} \quad \text { for every } t_{1}, t_{2} \in[0, T],
$$

where $\mathcal{G}_{\varepsilon}(u):=\int_{0}^{u}\left(1 / g_{\varepsilon}(y)\right) d y$. Observe, on the other hand, that

$$
\begin{aligned}
\left|\mathcal{G}_{\varepsilon}\left(x\left(t_{2}\right)\right)-\mathcal{G}_{\varepsilon}\left(x\left(t_{1}\right)\right)\right|= & \left|\int_{x\left(t_{1}\right)}^{x\left(t_{2}\right)} \frac{1}{g_{\varepsilon}(y)} d y\right| \\
& \geq \frac{1}{1+\varepsilon}\left|\int_{x\left(t_{1}\right)}^{x\left(t_{2}\right)} \frac{1}{g(y)} d y\right|=\frac{\left|\mathcal{G}\left(x\left(t_{2}\right)\right)-\mathcal{G}\left(x\left(t_{1}\right)\right)\right|}{1+\varepsilon},
\end{aligned}
$$


and hence,

$$
\left|\mathcal{G}\left(x\left(t_{2}\right)\right)-\mathcal{G}\left(x\left(t_{1}\right)\right)\right| \leq 2(1+\varepsilon)^{2}\|h\|_{L^{1}}, \quad t_{1}, t_{2} \in[0, T]
$$

Here, $\varepsilon>0$ is arbitrary, and letting $\varepsilon \rightarrow 0$ we get (3.2). This completes the proof of the lemma.

We consider next the 'potential energy' function

$$
\mathscr{G}(x):=-\int_{1}^{x} g(y) d y, \quad x>0 .
$$

Observe that it is strictly decreasing and convex. In this section we are assuming that the singularity of $g$ at $x=0$ is strong, and hence,

$$
\lim _{x \rightarrow 0} \mathscr{G}(x)=+\infty
$$

Let now $\left\{\bar{h}_{n}\right\}_{n}$ and $\left\{\rho_{n}\right\}_{n}$ be sequences of positive numbers with $\rho_{n} \rightarrow 0$ and $\bar{h}_{n} \mathscr{G}\left(\rho_{n}\right) \rightarrow+\infty$. Fix some compact interval $\left[t_{0}, t_{1}\right]$ and choose, for each positive integer $n$, a $W^{2,1}$ solution $\left.x_{n}:\left[t_{0}, t_{1}\right] \rightarrow\right] 0,+\infty$ [ of the initial value problem

$$
\left\{\begin{array}{l}
\ddot{x}_{n}(t) \geq \bar{h}_{n} g\left(x_{n}(t)\right), \quad t \in\left[t_{0}, t_{1}\right], \\
x_{n}\left(t_{0}\right)=\rho_{n}, \quad \dot{x}_{n}\left(t_{0}\right) \geq 0 .
\end{array}\right.
$$

Lemma 3.3. Under the above, $x_{n}(t) \rightarrow+\infty$ as $n \rightarrow \infty$, for any given $t \in$ $\left.t_{0}, t_{1}\right]$

Proof. There is no loss of generality in assuming that $x_{n}\left(t_{1}\right) \geq 1$ for each $n$ (otherwise we would replace $t_{1}$ by $t_{1}^{*}:=t_{1}+1$ and extend each function $x_{n}$ to $\left[t_{0}, t_{1}^{*}\right]$ by setting $x_{n}(t):=x_{n}\left(t_{1}\right)+\dot{x}_{n}\left(t_{1}\right)\left(t-t_{1}\right)+\left(1+\bar{h}_{n} g\left(x_{n}\left(t_{1}\right)\right)\right)\left(t-t_{1}\right)^{2}$ if $\left.\left.t \in] t_{1}, t_{1}+1\right]\right)$. Since each function $x_{n}$ is strictly convex, we see that, for $n$ big enough, there exists a unique number $\tau_{n} \in\left[t_{0}, t_{1}\right]$ such that $x_{n}\left(\tau_{n}\right)=1$.

It suffices to check that $\tau_{n} \rightarrow t_{0}$. With this aim we consider, for each $n \in \mathbb{N}$, the 'energy function'

$$
\left.\left.E_{n}(t):=\frac{\dot{x}_{n}(t)^{2}}{2}+\bar{h}_{n} \mathscr{G}\left(x_{n}(t)\right), \quad t \in\right] t_{0}, t_{1}\right]
$$

This is a $W^{1,1}$ function on $[0,+\infty[$ with nonnegative derivative. Hence,

$$
\left.\left.E_{n}(t) \geq E_{n}(0) \geq \bar{h}_{n} \mathscr{G}\left(\rho_{n}\right), \quad t \in\right] t_{0}, t_{1}\right],
$$

i.e. (since the derivatives $\dot{x}_{n}$ are nonnegative),

$$
\left.\left.\dot{x}_{n}(t) \geq \sqrt{2 \bar{h}_{n}} \sqrt{\mathscr{G}\left(\rho_{n}\right)-\mathscr{G}\left(x_{n}(t)\right)}, \quad t \in\right] t_{0}, t_{1}\right] .
$$


Divide both sides of this inequality by the right one and integrate between $t=t_{0}$ and $t=\tau_{n}$, to get

$$
\begin{aligned}
0 & \leq \tau_{n}-t_{0} \leq \frac{1}{\sqrt{2 \bar{h}_{n}}} \int_{t_{0}}^{\tau_{n}} \frac{\dot{x}_{n}(t)}{\sqrt{\mathscr{G}\left(\rho_{n}\right)-\mathscr{G}\left(x_{n}(t)\right)}} d t \\
& =\frac{1}{\sqrt{2 \bar{h}_{n}}} \int_{\rho_{n}}^{1} \frac{d y}{\sqrt{\mathscr{G}\left(\rho_{n}\right)-\mathscr{G}(y)}} d y=\frac{1}{\sqrt{2 \bar{h}_{n} \mathscr{G}\left(\rho_{n}\right)}} \int_{\rho_{n}}^{1} \frac{1}{\sqrt{1-\mathscr{G}(y) / \mathscr{G}\left(\rho_{n}\right)}} d y .
\end{aligned}
$$

By assumption, $\bar{h}_{n} \mathscr{G}\left(\rho_{n}\right) \rightarrow+\infty$, and hence, it remains only to show the boundedness of the sequence

$$
\left\{\int_{\rho_{n}}^{1} \frac{1}{\sqrt{1-\mathscr{G}(y) / \mathscr{G}\left(\rho_{n}\right)}} d y\right\}_{n} .
$$

With this goal we remember that the nonlinearity $g$ is decreasing, so that $\mathscr{G}$ is convex. Moreover, $\mathscr{G}(1)=0$, and consequently,

$$
\mathscr{G}(y) \leq \frac{\mathscr{G}\left(\rho_{n}\right)}{1-\rho_{n}}(1-y), \quad \text { if } y \in\left[\rho_{n}, 1\right],
$$

and hence,

$$
\frac{\mathscr{G}(y)}{\mathscr{G}\left(\rho_{n}\right)} \leq \frac{1-y}{1-\rho_{n}}, \quad y \in\left[\rho_{n}, 1\right]
$$

so that

$$
\int_{\rho_{n}}^{1} \frac{1}{\sqrt{1-\frac{\mathscr{G}(y)}{\mathscr{G}\left(\rho_{n}\right)}}} d y \leq \sqrt{1-\rho_{n}} \int_{\rho_{n}}^{1} \frac{1}{\sqrt{y-\rho_{n}}} d y=2\left(1-\rho_{n}\right) \rightarrow 2 \quad \text { as } n \rightarrow+\infty .
$$

The result follows.

We are immediately led to the following:

COROLLARY 3.4. Let the sequence $\left\{x_{n}\right\}_{n} \subset W^{2,1}\left(\left[t_{0}, t_{1}\right]\right)$ satisfy

$$
\min _{\left[t_{0}, t_{1}\right]} x_{n} \rightarrow 0, \quad \ddot{x}_{n}(t) \geq \bar{h} g\left(x_{n}(t)\right) \text { on }\left[t_{0}, t_{1}\right]
$$

for some constant $\bar{h}>0$. Then, $\max _{\left[t_{0}, t_{1}\right]} x_{n} \rightarrow+\infty$.

Proof. For any number $n$ we choose some number $\tau_{n} \in\left[t_{0}, t_{1}\right]$ where $x_{n}$ attains its minimum. If $\tau_{n} \leq\left(t_{0}+t_{1}\right) / 2$ we define $y_{n}:\left[0,\left(t_{0}+t_{1}\right) / 2\right] \rightarrow \mathbb{R}$ by $y_{n}(t):=x_{n}\left(\tau_{n}+t\right)$, while, if $\tau_{n} \geq\left(t_{0}+t_{1}\right) / 2$ we set $y_{n}:\left[0,\left(t_{0}+t_{1}\right) / 2\right] \rightarrow \mathbb{R}$ by $y_{n}(t):=x_{n}\left(\tau_{n}-t\right)$. The result follows by applying Lemma 3.3 to this new sequence. 


\section{The oscillation explodes as solutions approach the singularity}

In this final section we complete the proof of Theorem 1.1. Thus, we henceforth assume the framework of this result, i.e. that $\left(\mathrm{h}_{0}\right)-\left(\mathrm{h}_{2}\right)$ and $(\mathrm{g})$ hold; furthermore, we assume that either situation (A) or situation (B) hold. After introducing a translation of time we see that, for the sake of proving Theorem 1.1 there is no loss of generality in further assuming that there is some $\varepsilon>0$ such that

$$
h(t) \geq \bar{h}>0 \quad \text { if } t \in[0, \varepsilon] \cup[T-\varepsilon, T] .
$$

Combining Proposition 2.1 and Lemmas 2.3-3.2 with the comments preceding Lemma 3.2 we see that it will suffice to show the following:

Proposition 4.1. Under the assumptions above, let $\left\{x_{n}\right\}_{n} \subset W^{2,1}([0, T])$ be a sequence of solutions of (1.1) with $\min _{[0, T]} x_{n} \rightarrow 0$. Then, $\max _{[0, T]} x_{n} \rightarrow+\infty$.

The proof of Proposition 4.1 is especially simple in situation (A). Indeed, denoting by $\left.I_{1}=\right] 0, b_{1}\left[, I_{2}=\right] a_{2}, b_{2}\left[, \ldots, I_{p}=\right] a_{p}, T[$ the collection of (open) intervals where $h$ is constant and positive, there must exist one of them, say, $I_{k}$ such that $\min _{[0, T]} x_{n}=\min _{I_{k}} x_{n}$ for infinitely many indexes $n$. The result comes by applying Corollary 3.4 to the corresponding subsequence.

Thus, from now on we assume that situation (B) holds. Using a contradiction argument we further assume the existence of a sequence $x_{n}:[0, T] \rightarrow \mathbb{R}$ of solutions of (1.1) such that

$$
\min _{[0, T]} x_{n} \rightarrow 0 \text { but }\left\{\max _{[0, T]} x_{n}\right\}_{n} \text { is bounded. }
$$

We denote by $Z=\left\{b_{1}<a_{2}<b_{2}<\ldots<a_{p}\right\}$ the set of zeroes of $h$ in the time interval ] $0, T$, which are divided into two classes; the $a_{i}$ 's, where the derivative of $h$ is positive, and the $b_{i}$ 's, where the derivative of $h$ is negative. Observe also that, in view of (4.1), $h$ is positive on the set $I_{+}:=\left[0, b_{1}[\cup] a_{2}, b_{2}[\cup \ldots \cup\right.$ ]$\left.a_{p-1}, b_{p-1}[\cup] a_{p}, T\right]$, and negative on $\left.I_{-}:=\right] b_{1}, a_{2}[\cup \ldots \cup] b_{p-1}, a_{p}[$. We shall start our argument with the following:

LEMMA 4.2. $\min _{Z} x_{n} \rightarrow 0$.

Proof. We assume, on the contrary, that after passing to a subsequence there exists some constant $\delta_{0}>0$ such that $\min _{Z} x_{n} \geq \delta_{0}$ for every $n$. Let the sequence $\left\{\tau_{n}\right\}_{n} \subset[0, T]$ be defined by $x_{n}\left(\tau_{n}\right)=\min _{[0, T]} x_{n} \rightarrow 0$. Since all functions $x_{n}$ are strictly concave on each one of the intervals which compose $Z \cup I_{-}$, we see that $\tau_{n} \in I_{+}$for every $n \in \mathbb{N}$. Thus, after possibly passing to a subsequence, we may assume that $\tau_{n} \rightarrow \tau_{*} \in Z \cup I_{+}$. We distinguish two cases. 
Case 1. $\left[\tau_{*} \in I_{+}\right]$. Corollary 3.4 implies that $\max _{[0, T]} x_{n} \rightarrow+\infty$, contradicting the second part of (4.2).

Case 2. $\left[\tau_{*} \in Z\right]$. We apply the Mean Value Theorem to $x_{n}$ on the time interval $J_{n}:=\left[\min \left\{\tau_{n}, \tau_{*}\right\}, \max \left\{\tau_{n}, \tau_{*}\right\}\right]$, and obtain the existence of a sequence $c_{n} \rightarrow \tau_{*}$ with $c_{n} \in J_{n}$ for every $n$, such that $\sigma_{n} \dot{x}_{n}\left(c_{n}\right) \rightarrow+\infty$. Here, $\sigma_{n}=1$ if $\tau_{n}<c_{n}<\tau_{*}$ and $\sigma_{n}=-1$ if $\tau_{*}<c_{n}<\tau_{n}$. But $\dot{x}_{n}$ is increasing on $J_{n}$, and we deduce that $\left|\dot{x}_{n}\left(\tau_{*}\right)\right| \rightarrow+\infty$. Since $x_{n}\left(\tau_{*}\right) \geq \delta_{0}$ for every $n$ it is easy to check that $\max _{[0, T]} x_{n} \rightarrow+\infty$. This contradicts again the second part of (4.2) and concludes the proof of the lemma.

We continue now with the proof of Proposition 4.1 in situation (B). Since the set $Z$ is finite, after possibly passing to a subsequence we may assume the existence of a point $z_{*} \in Z$ such that $x_{n}\left(z_{*}\right) \rightarrow 0$. Two possibilities appear; either $z_{*} \in\left\{a_{2}, \ldots, a_{p}\right\}$ or $z_{*} \in\left\{b_{1}, \ldots, b_{p-1}\right\}$, but after possibly reversing the direction of time we may assume that $z_{*}=a_{k}$ for some $k \in\{2, \ldots, p\}$.

LEMma 4.3. $\limsup _{n \rightarrow+\infty} \dot{x}_{n}\left(a_{k}\right)<0$.

ProOF. Our first task will consist in observing that $\dot{x}_{n}\left(a_{k}\right)<0$ provided that $n$ is big enough. We use a contradiction argument and assume instead that, after possibly passing to a subsequence, one has $\dot{x}_{n}\left(a_{k}\right) \geq 0$ for every $n$. Since $x_{n}$ is concave on $\left[b_{k-1}, a_{k}\right]$, it follows that $0<x_{n}(t) \leq x_{n}\left(a_{k}\right)$ and $\ddot{x}_{n}(t) \leq h(t) g\left(x_{n}\left(a_{k}\right)\right)$ on $\left[b_{k-1}, a_{k}\right]$, and, integrating twice this inequality,

$x_{n}\left(b_{k-1}\right) \leq x_{n}\left(a_{k}\right)-\dot{x}_{n}\left(a_{k}\right)\left(a_{k}-b_{k-1}\right)+g\left(x_{n}\left(a_{k}\right)\right) \int_{b_{k-1}}^{a_{k}}\left(t-b_{k-1}\right) h(t) d t \rightarrow-\infty$ as $n \rightarrow+\infty$. This is a contradiction because all functions $x_{n}$ are positive.

Using again a contradiction argument, assume now that $\dot{x}_{n}\left(a_{k}\right)<0$ for every $n$ but $\dot{x}_{n}\left(a_{k}\right) \rightarrow 0$. Since $x_{n}$ is concave on $\left[b_{k-1}, a_{k}\right]$, it follows that $0<x_{n}(t) \leq \rho_{n}:=x_{n}\left(a_{k}\right)-\left(a_{k}-b_{k-1}\right) \dot{x}_{n}\left(a_{k}\right)$ for every $t \in\left[b_{k-1}, a_{k}\right]$ and $\ddot{x}_{n}(t) \leq h(t) g\left(\rho_{n}\right)$ on $\left[b_{k-1}, a_{k}\right]$. Arguing as before, we deduce

$$
x_{n}\left(b_{k-1}\right) \leq x_{n}\left(a_{k}\right)-\dot{x}_{n}\left(a_{k}\right)\left(a_{k}-b_{k-1}\right)+g\left(\rho_{n}\right) \int_{b_{k-1}}^{a_{k}}\left(t-b_{k-1}\right) h(t) d t \rightarrow-\infty
$$

as $n \rightarrow+\infty$, since $\rho_{n} \rightarrow 0$. This is again a contradiction and concludes the proof.

Since $h$ is positive on $] a_{k}, b_{k}$ [, all functions $x_{n}$ are strictly convex on the closed interval $\left[a_{k}, b_{k}\right]$. Our next result gives some further information on these functions.

Lemma 4.4. For $n$ big enough there is an unique point $\left.t_{n} \in\right] a_{k}, b_{k}[$ such that $\dot{x}_{n}\left(t_{n}\right)=0$. Moreover, $t_{n} \rightarrow a_{k}$ as $n \rightarrow+\infty$. 
Proof. The uniqueness of the critical point is immediate from the convexity of $x_{n}$ on $\left[a_{k}, b_{k}\right]$. Thus, using a contradiction argument, we assume that, after possibly passing to a subsequence, there exists some $0<\tau_{*}<b_{k}-a_{k}$ such that $\dot{x}_{n}(t)<0$ for every $t \in\left[a_{k}, a_{k}+\tau_{*}\right]$ and every $n \in \mathbb{N}$. Applying the Mean Value Theorem to the function $x_{n}$ on the time interval $\left[a_{k}, a_{k}+\tau_{*} / 2\right]$ we obtain the existence of a sequence $a_{k}<r_{n}<a_{k}+\tau_{*}$ such that $\dot{x}_{n}\left(r_{n}\right) \rightarrow 0$. But the functions $\dot{x}_{n}$ are all of them increasing on $\left[a_{k}, b_{k}\right]$, and hence, both sequences $\left\{\dot{x}_{n}\left(a_{k}+\tau_{*} / 2\right)\right\}_{n}$ and $\left\{\dot{x}_{n}\left(a_{k}+\tau_{*}\right)\right\}_{n}$ converge to 0 . On the other hand, $x_{n}(t) \leq x_{n}\left(a_{k}\right)$ for every $t \in\left[a_{k}, a_{k}+\tau_{*}\right]$, and we deduce

$\dot{x}_{n}\left(a_{k}+\tau_{*}\right)-\dot{x}_{n}\left(a_{k}+\tau_{*} / 2\right)=\int_{\tau_{*} / 2}^{\tau_{*}} h(t) g\left(x_{n}(t)\right) d t \geq g\left(x_{n}\left(a_{k}\right)\right) \int_{\tau_{*} / 2}^{\tau_{*}} h(t) d t \rightarrow+\infty$ as $n \rightarrow+\infty$, a contradiction. The proof is complete.

We find again two possibilities: either $\dot{x}_{n}\left(a_{k}\right) \rightarrow-\infty$ or we may assume, after possibly passing to a subsequence, that $\dot{x}_{n}\left(a_{k}\right) \rightarrow-v<0$. We conclude the proof of Proposition 4.1 by studying both cases separately.

Proof of Proposition 4.1.

Case 1. $\left[\dot{x}_{n}\left(a_{k}\right) \rightarrow-\infty\right.$ as $n \rightarrow+\infty$. Fix some point $\left.t_{*} \in\right] a_{k}, b_{k}[$. We recall that $h$ is continuously differentiable around its zero $a_{k}$ to find some $0<\varepsilon<t_{*}-a_{k}$ such that, for every $\left.t \in] a_{k}, a_{k}+\varepsilon\right]$,

$$
\left.0<h(s)<h(t), \quad \text { if } s \in] a_{k}, t[, \quad h(t)<h(s), \quad \text { if } s \in] t, t_{*}\right] .
$$

For $n$ big enough, $x_{n}\left(a_{k}\right)<1$ and the points $\left.t_{n} \in\right] a_{k}, b_{k}$ [ given by Lemma 4.4 satisfy $0<t_{n}-a_{k}<\varepsilon<t_{*}-t_{n}$. We consider the function $E_{n}:\left[a_{k}, t_{n}\right] \rightarrow \mathbb{R}$ defined by

$$
E_{n}(t):=\frac{1}{2} \dot{x}_{n}(t)^{2}+h\left(t_{n}\right) \mathscr{G}\left(x_{n}(t)\right),
$$

where $\mathscr{G}$ is given by (3.6). Direct differentiation shows that $E_{n}$ is increasing on its domain, and hence,

$$
E_{n}\left(t_{n}\right)=h\left(t_{n}\right) \mathscr{G}\left(x_{n}\left(t_{n}\right)\right) \geq E_{n}\left(a_{k}\right) \geq \dot{x}_{n}\left(a_{k}\right)^{2} / 2 \rightarrow+\infty \quad \text { as } n \rightarrow+\infty,
$$

the second inequality follows from the fact that $\mathscr{G}(y)>0$ if $y<1$. Applying Lemma 3.3 to the functions $t \mapsto x_{n}\left(t_{n}+t\right)$ on the interval $\left[t_{0}, t_{1}\right]=[0, \varepsilon]$, we find that $x_{n}\left(t_{n}+\varepsilon\right) \rightarrow+\infty$, contradicting the second part of (4.2).

Case 2. $\dot{x}_{n}\left(a_{k}\right) \rightarrow-v<0$.

Fix some point $\left.t_{*} \in\right] b_{k-1}, a_{k}$; being concave on $\left[t_{*}, a_{k}\right]$, all functions $x_{n}$ lie below their tangent line at $t=a_{k}$, i.e.

$$
x_{n}(t) \leq x_{n}\left(a_{k}\right)+\left(t-a_{k}\right) \dot{x}_{n}\left(a_{k}\right), \quad t \in\left[t_{*}, a_{k}\right] .
$$


In particular, $x_{n}\left(t_{*}\right) \leq x_{n}\left(a_{k}\right)-\left(a_{k}-t_{*}\right) \dot{x}_{n}\left(a_{k}\right)$, and the sequence $\left\{x_{n}\left(t_{*}\right)\right\}_{n}$ is bounded. Moreover,

$$
\begin{array}{rl}
\dot{x}_{n}\left(t_{*}\right)=\dot{x}_{n}\left(a_{k}\right)-\int_{t_{*}}^{a_{k}} & h(t) g\left(x_{n}(t)\right) d t \\
& \geq \dot{x}_{n}\left(a_{k}\right)-\int_{t_{*}}^{a_{k}} h(t) g\left(x_{n}\left(a_{k}\right)-\left(a_{k}-t\right) \dot{x}_{n}\left(a_{k}\right)\right) d t,
\end{array}
$$

and, by Fatou's Lemma,

$$
\liminf _{n \rightarrow+\infty} \dot{x}_{n}\left(t_{*}\right) \geq-v-\int_{t_{*}}^{a_{k}} h(t) g\left(\left(a_{k}-t\right) v\right) d t=+\infty
$$

the last equality arising from the fact that, being in situation (B), $a_{k}$ is a nondegenerate zero of $h$ and the singularity of $g$ at $x=0$ is very strong. Thus, $\dot{x}_{n}\left(t_{*}\right) \rightarrow+\infty$, and since $\left\{x_{n}\left(t_{*}\right)\right\}_{n}$ is bounded and the functions $x_{n}$ are concave on $\left[b_{k-1}, t_{*}\right]$ we deduce that they all vanish somewhere on this interval, at least for big $n$. This is impossible and concludes the proof.

\section{REFERENCES}

[1] R. Agarwal and D. O'Regan, A Survey of recent results for initial and boundary value problems singular in the dependent variable, Handbook of Differential Equations, 1-68, Elsevier/North-Holland, Amsterdam, 2004.

[2] L.E. Bobisud, D. O'Regan and W.D. Royalty, Solvability of some nonlinear boundary value problems, Nonlinear Anal. 12 (1988), no. 9, 855-869.

[3] A. Boscaggin And F. Zanolin, Second order ordinary differential equations with indefinite weight: the Neumann boundary value problem, Preprint, 2013.

[4] J.L. Bravo and P.J. Torres, Periodic solutions of a singular equation with indefinite weight, Adv. Nonlinear Stud. 10 (2010), no. 4, 927-938.

[5] E.N. DANCER, On the use of asymptotics in nonlinear boundary value problems, Ann. Mat. Pura Appl. (4) 131 (1982), 167-185.

[6] C. De Coster and P. Habets, Two-Point Boundary Value Problems: Lower and Upper Solutions, Mathematics in Science and Engineering, 205, Elsevier B.V., Amsterdam, 2006.

[7] J.A. Gatica, V. Oliker And P. Waltman, Singular nonlinear boundary value problems for second-order ordinary differential equations J. Differential Equations 79 (1989), no. 1, $62-78$.

[8] M. Gaudenzi, P. Habets and F. Zanolin, Positive solutions of singular boundary value problems with indefinite weight, Bull. Belg. Math. Soc. 9 (2002), 607-619.

[9] W.B. Gordon, Conservative dynamical systems involving strong forces, Trans. Amer. Math. Soc. 204 (1975), 113-135.

[10] Z. Guo, Solvability of some singular nonlinear boundary value problems and existence of positive radial solutions of some nonlinear elliptic problems, Nonlinear Anal. 16 (1991), no. 9, 781-790.

[11] P. Habets And F. Zanolin, Upper and lower solutions for a generalized Emden-Fowler equation, J. Math. Anal. Appl. 181 (1994), no. 3, 684-700.

[12] J. JANus AND J. MYJAK, A generalized Emden-Fowler equation with a negative exponent, Nonlinear Anal. 23 (1994), no. 8, 953-970. 
[13] C.D. Luning And W.L. PERry, Positive solutions of negative exponent generalized Emden-Fowler boundary value problems, SIAM J. Math. Anal. 12 (1981), no. 6, 874879 .

[14] A. Nachman And A. Callegari, A nonlinear singular boundary value problem in the theory of pseudoplastic fluids, SIAM J. Appl. Math. 38 (1980), no. 2, 275-281.

[15] A.J. UREÑA, Some counterexamples for singular equations with indefinite weight, in preparation.

[16] S.D. Taliaferro, A nonlinear singular boundary value problem, Nonlinear Anal. 3 (1979), no. $6,897-904$.

Manuscript received June 27, 2014 accepted October 15, 2014

Antonio J. UREÑA

Departamento de Matemática Aplicada

Universidad de Granada

Granada, 18071, SPAIN

E-mail address: ajurena@ugr.es 